\title{
Autoimmunity risk alleles: hotspots in B cell regulatory signaling pathways
}

\author{
John C. Cambier
}

Integrated Department of Immunology, University of Colorado School of Medicine and National Jewish Health, Denver, Colorado, USA.

\begin{abstract}
Autoimmunity is the consequence of the combination of genetic predisposition and environmental effects, such as infection, injury, and constitution of the gut microbiome. In this edition of the JCI, Dai et al. describe the use of knockin technology to test the mechanism of action of a polymorphism in the protein tyrosine phosphatase nonreceptor 22 (PTPN22) (LYP) that is associated with susceptibility to multiple autoimmune diseases. The function of this allele, and that of a disproportionate number of autoimmune disease risk alleles, suggests that inhibitory signaling pathways that maintain B lymphocyte immune tolerance may represent an Achilles' heel in the prevention of autoimmunity.
\end{abstract}

\section{Of immunologic tolerance, B cells, and autoimmunity}

The immune system is charged with protecting us from the myriad pathogens in our environment and has the added chore of recognizing and eliminating rogue cells that threaten to cause cancer. Competence to generate immune responses is dependent in part on continued generation of new lymphocytes, $T$ cells and $B$ cells, each expressing receptors with unique specificity for antigen. Indeed, we each produce in excess of 10 billion lymphocytes each day. Just by chance, most of these newly produced lymphocytes recognize self antigens and must be silenced to prevent the generation of an immune response to self and resultant autoimmunity (1). Silencing of autoreactive lymphocytes leads to a state of unresponsiveness called immunological tolerance. SLE is an autoimmune disease that usually afflicts women and is caused by loss of immunological tolerance to nucleic acids and their associated proteins, resulting in the production of pathogenic autoantibodies that damage tissues and cause inflammation. A major thrust of contemporary biomedical science is to understand how and why immune tolerance fails in some individuals, thus leading to autoimmune diseases such as SLE.

It is clear that development of autoimmunity is determined by both nature and nurture. Susceptibility is affected by genet-

Conflict of interest: The author has declared that no conflict of interest exists.

Citation for this article: J Clin Invest. 2013; 123(5):1928-1931. doi:10.1172/JCI69289. ics as well as personal experience, such as history of infection and injury. As recently as 2008, only a handful of genetic polymorphisms that confer risk of SLE were recognized, and these had been identified based on candidate gene approaches. With the development of high-throughput genomewide analysis of SNPs has come a torrent of new information that has the potential to provide a much more comprehensive view of disease-associated variants. In circumstances in which the polymorphism is found in a structural gene or its regulatory regions, genome-wide association studies (GWAS) can implicate a specific protein and physiologic processes in disease. GWAS have expanded the list of genetic polymorphisms that confer risk of development of SLE to more than 25; most of these affect proteins involved in specific immune functions, particularly interferon signaling, antigen presentation, and lymphocyte antigen receptor signaling (2-6).

Studies of Dai et al. (7) in this issue of the JCI stand as testimony to the power of using complementary approaches: combining the identification of disease risk alleles with studies in mice that define the altered function of proteins encoded by risk alleles. Based on previous demonstrations of association of a specific allele of the phosphotyrosine phosphatase PTPN22 with risk of developing multiple autoimmune diseases, including SLE, RA, type 1 diabetes (T1D), and others (8-11), Dai et al. created and studied mice in which a mutation, R619W, analogous to the variance found in the human risk allele (R620W) (12) was knocked into the murine ortholog of the gene. The authors characterized the effect of the mutation on lymphocyte signaling and various immunological parameters. The results provide important new insight regarding the role of PTPN22 in regulation of signal transduction by antigen receptors and thus in maintenance of immune tolerance. Dai et al. found that the R619W variance did not affect protein half-life of PTPN22 as previously suggested (13), but rather altered receptor-mediated protein tyrosine phosphorylation and caused hypersensitivity to antigen receptor stimulation. Furthermore, B cell expression of the variant was sufficient to promote autoimmunity. Thus, PTPN22 is among the SLE risk alleles defined thus far that result in compromised control of intracellular BCR signaling, leading to loss of B cell tolerance and autoimmunity (2-7). It may be telling that genes encoding molecules that enforce B cell tolerance are disproportionately represented among risk alleles. These findings suggest that autoreactive B cells may be particularly important in the initiation of autoimmunity and that regulation of antigen receptor signaling pathways is fragile yet critical to preventing immune responses to autoantigens.

\section{Anergic B cells, an Achilles' heel of immune tolerance}

Available evidence indicates that newly produced autoreactive $B$ cells are silenced by three mechanisms $(1,14,15)$. Those reactive with high-avidity antigens, such as cell-surface molecules, are induced by resultant strong $B$ cell antigen receptor (BCR) signals to edit antigen receptor specificity, a mechanism called receptor editing. If editing fails to eliminate autoreactivity, these cells are induced by autoantigen to die, which is referred to as clonal deletion. Editing and clonal deletion are thought to occur primarily in the bone marrow where the cells are produced. $\mathrm{B}$ cells that recognize low-avidity self antigens, such as soluble proteins, proceed through development and can be found in peripheral lymphoid organs such as spleen and lymph nodes as well as 


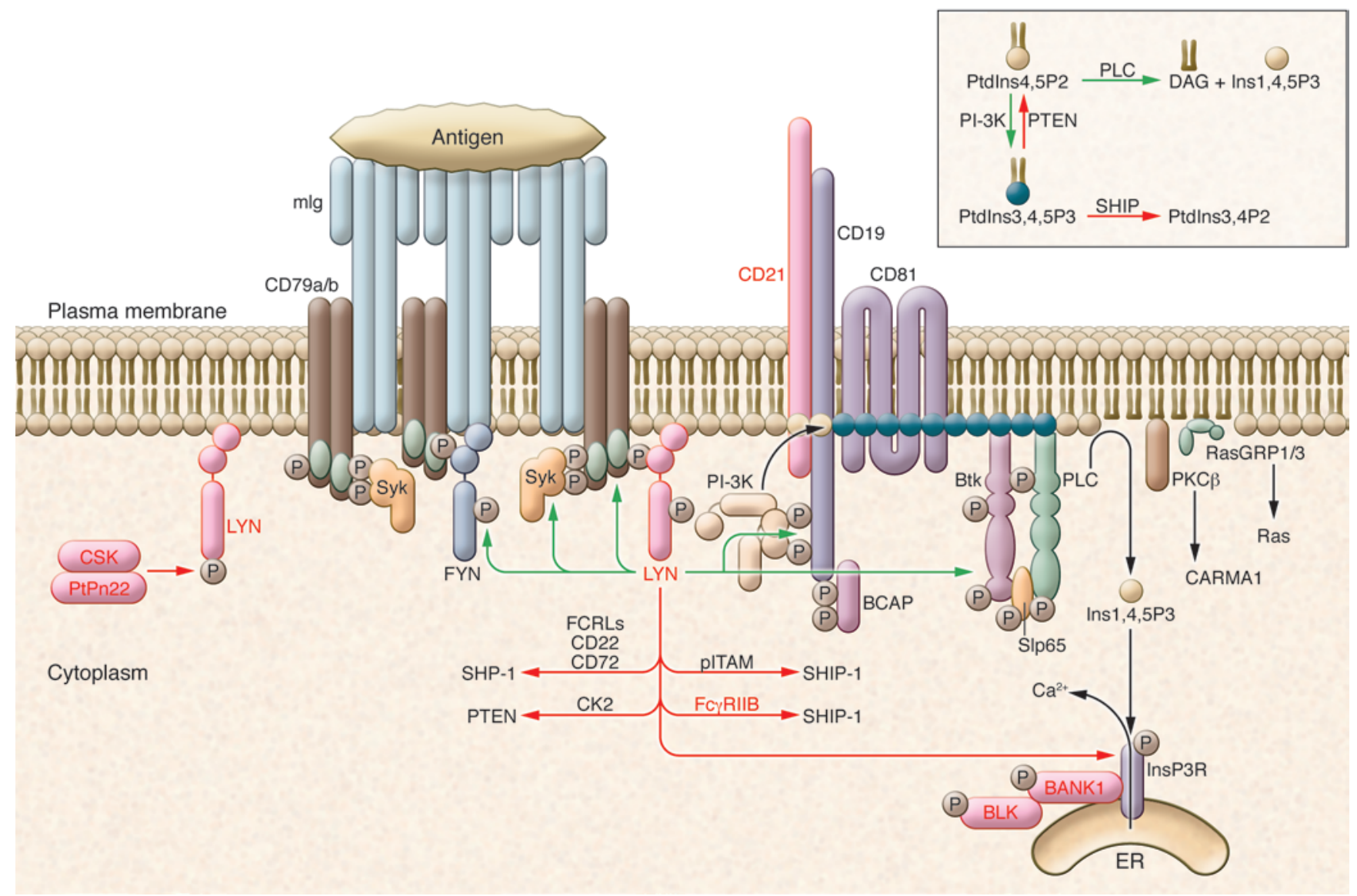

\section{Figure 1}

Proximal activating and regulatory signal transduction by B lymphocyte antigen receptors. Antigen aggregation of receptors composed of membrane $\mathrm{lg}(\mathrm{mlg})$ and transducers CD79a and CD79b leads to tyrosine phosphorylation of CD79 immunoreceptor tyrosine-based activation motifs (ITAMs) (green) by Lyn/Fyn Src family tyrosine kinases. Participation of Src family kinases in this signaling pathway is negatively regulated by CSK-mediated phosphorylation of their C-terminal tyrosines. Phosphorylation of conserved ITAM motif tyrosines leads to recruitment of SH2 domain-containing effectors, including Syk, Lyn, and Fyn, and further activation of these effectors, initiating downstream pathways leading to cell activating (green arrows) and opposing regulatory signaling (red arrows). Among these proximally acting kinases, Lyn uniquely initiates both activating and regulatory circuitry by phosphorylation of immunoreceptor tyrosine-based inhibitory motifs (ITIMs) embedded in cytoplasmic tails of membrane proteins, including FCRLs, CD72, CD22, FcyRIIB, and other molecules. These phosphorylated motifs propagate inhibitory signals via activation of SHP and SHIP phosphatases. Lyn may also act through other intermediaries, e.g., monophosphorylated antigen receptor ITAMs, casein kinase 2, and components of the BLK/BANK1/InsP3 receptor complex on ER membranes, to trigger regulatory circuitry. Shown in red lettering are molecules in which certain allelic forms have been shown to increase risk of development of SLE.

in blood and lymph and at other sites. They continue to express antigen receptors and, while many of these can be unoccupied, they are unresponsive to stimulation by crossreactive environmental antigens that essentially mimic the autoantigen. This mechanism of autoreactive B cell silencing is termed anergy. Antigen unresponsiveness of anergic cells is the consequence of chronic antigen receptor signaling, which leads to nondurable changes in receptor coupling to downstream biochemical pathways. While the initial B cell encounter with antigen leads to the biased activation of pathways leading to cell activation, chronically stimulated cells respond by biased activation of feedback regulatory pathways, preventing further activation. Because they occur in peripheral tissues, anergic B cells are more likely than autoreactive cells in the bone marrow to be exposed to locally generated inflammatory mediators. These environmental signals may conspire with risk-conferring genetic polymorphisms to ultimately cause autoimmunity.

\section{Propagation of opposing signals by $B$ cell antigen receptors}

$B$ cell antigen receptor signaling is induced by aggregation of membrane immunoglobulins and associated transducer chains CD79a and CD79b (Figure 1). The cytoplasmic tails of CD79 function as the nexus for recruitment of downstream signaling pathways. Phosphorylation of conserved CD79 tyrosine residues by SRC and Syk family tyrosine kinases facilitates $\mathrm{SH} 2$ domain-mediated binding and activation of effectors including these kinases themselves. Although the predominant SRC family kinase expressed by B cells is Lyn, these cells also express Blk and variable levels of Fyn. While Blk appears restricted in subcellular distribution primarily to cytoplasmic organelles (13) and thus may not participate in initial signaling events, both Lyn and Fyn are able to initiate BCR transduction of activating signals (16). 
Lyn appears unique among SRC family kinases in its ability to engage not only activating signaling circuitry, but also the inhibitory circuitry that keeps anergic B cells in check (17). Lyn-knockout mice develop severe lupus-like disease consistent with lack of regulatory signaling. On the other hand, Blk- and Fyn-knockout mice do not develop autoimmunity (18, 19). However, BLK is implicated in negative regulation of BCR signaling $(18,19)$. Lyn mediates its inhibitory effects by phosphorylation of plasma membrane receptors such as CD22, CD72, and FcyRIIB, which act in turn by recruiting protein and inositol phosphatases (SHP-1 and SHIP-1, respectively) (20). The activity of these enzymes can also be regulated by Lynmediated phosphorylation. While SHP-1 mediates dephosphorylation of ITAMs and other proximal intermediaries in activating signaling pathways, SHIP-1 degrades PtdIns3,4,5P3 needed for activation of Akt, phospholipase $\mathrm{C}$, and calcium signaling. Furthermore, PtdIns3,4P2 generated by SHIP-1 recruits the inhibitory adaptor proteins TAPP1 and TAPP2. Mice with B celltargeted knockout of SHP-1 (21) or SHIP-1 (22) as well as knockins of inactive TAPPs develop severe lupus-like disease (23). Lyn may also mediate inhibitory signaling via activation of cytosolic mediators such as casein kinase 2 , which by phosphorylation of the tail of phosphatase and tensin homolog (PTEN) extends its half-life, promoting its inhibitory functions (24). PTEN expression appears important in maintenance of anergy (25). Finally, although less clear mechanistically, Lyn phosphorylates an ER-associated protein complex composed of BLK, BANK1, and the inositol triphosphate receptors (InsP3R), thereby regulating $\mathrm{Ca}^{+2}$ release from intracellular stores $(13,26)$.

Adding additional complexity, participation of Lyn (and other SRC family kinases) in antigen receptor signaling is regulated by phosphorylation of a tyrosine residue located near the $\mathrm{C}$ terminus of the molecule. When phosphorylated by CSK (c-SRC kinase), this phosphotyrosine mediates an intramolecular interaction with the SH2 domain, repressing enzymatic activity and preventing recruitment of the kinase to phosphorylated ITAMs.

\section{SLE susceptibility alleles that affect regulatory signaling in $B$ cells}

Genetic polymorphisms affecting expression levels or function of the seven pro- teins annotated in Figure 1 are associated with SLE. A SNP in the promoter region of the CSK gene is associated with increased expression of CSK (5). Overexpression of CSK, a negative regulator of Lyn, would reduce the activity of inhibitory circuitry downstream from Lyn. PTPN22, a CSKassociated phosphatase, may somehow impinge on this phosphorylation, but based on the work of Dai et al., the risk allele also affects the spectrum of proteins phosphorylated upon antigen receptor stimulation (3). It has been reported that B cells in $66 \%$ of SLE patients exhibit reduced Lyn expression (4). While the genetic basis of this is unclear, reduced Lyn expression, like increased CSK expression, would be expected to reduce receptor activation of inhibitory signaling. An allele of CR2, the type 2 complement receptor, containing a 5' UTR polymorphism that determines reduced expression, increases SLE risk (2). CR2 is a member of a B cell membrane protein complex containing CD19. CD19 is a substrate of Lyn and is involved in PI3K activation, and CD19 also acts in the processive activation of Lyn (27). Decreased CD21 expression may therefore compromise regulatory Lyn signaling stimulated by opsonized antigens.

Polymorphisms in genes encoding three Lyn substrates also contribute to SLE risk. Risk alleles of the gene for the inhibitory receptor for IgG (FcyRIIB) encode receptors defective in signal transduction (7). As a consequence of this mutation, B cells are not subject to efficient regulation by IgGcontaining immune complexes. Another risk allele promotes alternate splicing of $B L K$ mRNA, resulting in reduced BLK expression and, presumably, regulation of its binding partners BANK1 and the InsP3 receptor. BLK haploinsufficiency in the mouse leads to autoimmunity and hypersensitivity to antigen receptor stimulation (18). Finally, a BANK1 risk allele may alter regulation of calcium mobilization following BCR stimulation. Thus, polymorphisms in CSK, PTPN22, LYN, Fc $\gamma$ RIIB, BLK, and BANK1 may all compromise $B$ cell tolerance by impairing regulation of antigen receptor signaling.

\section{Conclusions}

SLE is an autoimmunity mediated by autoantibodies produced by descendants of autoreactive B lymphocytes. While in healthy individuals, these $B$ cells are silenced by regulatory signaling pathways, studies in mice demonstrate that failure of these mechanisms confers risk of disease development. Perhaps it should come as no surprise that some human SLE risk alleles, such as PTPN22R620W, have an impact on these signaling pathways. However, considering the myriad cells and processes that impinge on the immune response, it is surprising that such a large proportion of risk alleles defined to date are operative in regulation of $B$ cell antigen receptor signaling. It will be interesting to see whether this trend continues as GWAS define new susceptibility alleles. These findings both underscore the importance of regulation of BCR signaling in preventing autoimmunity and indicate that operative mechanisms are finely tuned and fragile. Finally, they may reveal fruitful targets for disease prevention and therapy.

\section{Acknowledgments}

The author acknowledges research support from the NIH and the Juvenile Diabetes Research Foundation.

Address correspondence to: John C. Cambier, Ida and Cecil Green Distinguished Professor and Chair, Integrated Department of Immunology, University of Colorado School of Medicine and National Jewish Health, 1400 Jackson St., Denver, Colorado 80206, USA. Phone: 303.398.1325; Fax: 303.270.2325; E-mail: cambierj@njhealth.org.

1. Wardemann H, Nussenzweig MC. B-cell self-tolerance in humans. Adv Immunol. 2007;95:83-110.

2. Cruickshank MN, et al. Transcriptional effects of a lupus-associated polymorphism in the 5 ' untranslated region (UTR) of human complement receptor 2 (CR2/CD21). Mol Immunol. 2012;52(3-4):165-173.

3. Kono H, et al. FcgammaRIIB Ile232Thr transmembrane polymorphism associated with human systemic lupus erythematosus decreases affinity to lipid rafts and attenuates inhibitory effects on B cell receptor signaling. Hum Mol Genet. 2005;14(19):2881-2892.

4. Liossis SN, Solomou EE, Dimopoulos MA, Panayiotidis P, Mavrikakis MM, Sfikakis PP. B-cell kinase lyn deficiency in patients with systemic lupus erythematosus. J Investig Med. 2001;49(2):157-165.

5. Manjarrez-Orduno N, et al. CSK regulatory polymorphism is associated with systemic lupus erythematosus and influences B-cell signaling and activation. Nat Genet. 2012;44(11):1227-1230.

6. Taylor KE, et al. Risk alleles for systemic lupus erythematosus in a large case-control collection and associations with clinical subphenotypes. PLoS Genet. 2011;7(2):e1001311

7. Dai X, et al. A disease-associated PTPN22 variant promotes systemic autoimmunity in murine models. J Clin Invest. 2013;123(5):2024-2036.

8. Bottini $\mathrm{N}$, et al. A functional variant of lymphoid tyrosine phosphatase is associated with type I diabetes. Nat Genet. 2004;36(4):337-338.

9. Bottini N, Vang T, Cucca F, Mustelin T. Role of PTPN22 in type 1 diabetes and other autoimmune diseases. Semin Immunol. 2006;18(4):207-213.

10. Chung SA, Criswell LA. PTPN22: its role in SLE and 
autoimmunity. Autoimmunity. 2007;40(8):582-590.

11. Vang T, Miletic AV, Arimura Y, Tautz L, Rickert RC, Mustelin T. Protein tyrosine phosphatases in autoimmunity. Annu Rev Immunol. 2008;26:29-55.

12. Vang T, et al. Autoimmune-associated lymphoid tyrosine phosphatase is a gain-of-function variant. Nat Genet. 2005;37(12):1317-1319.

13. Zhang J, et al. The autoimmune disease-associated PTPN22 variant promotes calpain-mediated Lyp/Pep degradation associated with lymphocyte and dendritic cell hyperresponsiveness. Nat Genet. 2011;43(9):902-907.

14. Cambier JC, Gauld SB, Merrell KT, Vilen BJ. $\mathrm{B}$-cell anergy: from transgenic models to naturally occurring anergic B cells? Nat Rev Immunol. 2007;7(8):633-643

15. Pelanda R, Torres RM. Receptor editing for better or for worse. Curr Opin Immunol. 2006;18(2):184-190.

16. Pelanda R, Torres RM. Central B-cell tolerance: where selection begins. Cold Spring Harb Perspect
Biol. 2012;4(4):a007146.

17. Horikawa K, Nishizumi H, Umemori H, Aizawa S, Takatsu K, Yamamoto T. Distinctive roles of Fyn and Lyn in IgD- and IgM-mediated signaling. Int Immunol. 1999;11(9):1441-1449.

18. DeFranco AL, Chan VW, Lowell CA. Positive and negative roles of the tyrosine kinase Lyn in B cell function. Semin Immunol. 1998;10(4):299-307.

19. Castillejo-Lopez C, et al. Genetic and physical interaction of the B-cell systemic lupus erythematosus-associated genes BANK1 and BLK. Ann Rheum Dis. 2012;71(1):136-142.

20. Ravetch JV, Lanier LL. Immune inhibitory receptors. Science. 2000;290(5489):84-89.

21. Pao LI, et al. B cell-specific deletion of protein-tyrosine phosphatase Shp1 promotes B-1a cell development and causes systemic autoimmunity. Immunity. 2007;27(1):35-48.

22. O'Neill SK, et al. Monophosphorylation of CD79a and CD79b ITAM motifs initiates a SHIP-1 phosphatase- mediated inhibitory signaling cascade required for B cell anergy. Immunity. 2011;35(5):746-756.

23. Landego I, et al. Interaction of TAPP adapter proteins with phosphatidylinositol $(3,4)$-bisphosphate regulates B-cell activation and autoantibody production. Eur J Immunol. 2012;42(10):2760-2770.

24. Song MS, Salmena L, Pandolfi PP. The functions and regulation of the PTEN tumour suppressor. Nat Rev Mol Cell Biol. 2012;13(5):283-296.

25. Browne CD, Del Nagro CJ, Cato MH, Dengler HS, Rickert RC. Suppression of phosphatidylinositol 3,4,5-trisphosphate production is a key determinant of B cell anergy. Immunity. 2009;31(5):749-760.

26. Yokoyama K, et al. BANK regulates BCR-induced calcium mobilization by promoting tyrosine phosphorylation of IP(3) receptor. EMBOJ. 2002;21(1-2):83-92.

27. Fujimoto M, et al. CD19 regulates Src family protein tyrosine kinase activation in B lymphocytes through processive amplification. Immunity. 2000; 13(1):47-57.

\title{
Illuminating regeneration: noninvasive imaging of disease progression in muscular dystrophy
}

\author{
Jennifer R. Levy and Kevin P. Campbell \\ Howard Hughes Medical Institute, Department of Molecular Physiology and Biophysics, Department of Neurology, Department of Internal Medicine, \\ Roy J. and Lucille A. Carver College of Medicine, The University of lowa, lowa City, lowa, USA.
}

\begin{abstract}
Muscular dystrophies are characterized by progressive muscle weakness and wasting. Among the key obstacles to the development of therapies is the absence of an assay to monitor disease progression in live animals. In this issue of the JCI, Maguire and colleagues use noninvasive bioluminescence imaging to monitor luciferase activity in mice expressing an inducible luciferase reporter gene in satellite cells. These cells proliferate in response to degeneration, therefore increasing the level of luciferase expression in dystrophic muscle.
\end{abstract}

\section{Introduction}

Skeletal muscle has a robust regenerative capacity, with rapid reestablishment of full strength, even after severe damage to the tissue. Regeneration is mediated by muscle stem cells, called satellite cells. In response to muscle damage, satellite cells proliferate, differentiate into myoblasts, and fuse into myotubes, which act to repair damaged muscle. In muscular dystrophies, continuous muscle degeneration is accompanied by regeneration of muscle fibers mediated by satellite cell progeny (1).

Currently, the standard method for evaluating disease progression in muscular dystrophy animal models is muscle histopathology. This approach is labor inten-

Conflict of interest: The authors have declared that no conflict of interest exists.

Citation for this article: J Clin Invest. 2013; 123(5):1931-1934. doi:10.1172/JCI69568. sive, as it involves the removal and processing of the tissue of interest, imaging of the slides, and analysis of the images. Furthermore, the invasiveness of this approach does not permit consecutive sampling, hindering the ability to evaluate the course of a disease or success of a therapeutic strategy. Other methods for evaluating muscle disease include behavior testing and force testing of the dissected muscle, although the specificity of the results obtained from these tests can often be difficult to assess. High levels of serum biomarkers, such as serum creatine kinase, can be indicative of muscle damage, but levels depend on muscle mass and can be widely variable over time in individual dystrophic mice (2).

Perhaps the best candidate technology for studying muscle disease in live animals is MRI, which can reveal the permeability of muscle fibers correlating with disease severity (3). While MRI is noninvasive, it is more expensive and less widely available than bioluminescence imaging systems in animal research laboratories.

\section{A "regeneration reporter" mouse strain}

The first group to use bioluminescence imaging to reveal satellite cell proliferation was Sacco et al., who transplanted a single luciferase-expressing satellite cell into the tibialis anterior (TA) muscle of NOD/SCID mice that were depleted of endogenous satellite cells by irradiation (4). They observed that a single luciferase-expressing satellite cell is capable of self renewal after transplantation. Further, they found a substantial increase in satellite cell proliferation, as indicated by increased bioluminescence values, in response to muscle tissue damage by notexin.

In this issue, Maguire et al. (5) utilized the Pax7Cre ${ }^{E R} /$ LuSEAP mouse first generated by Nishijo et al. (6) to develop a mouse model that could be used to monitor muscle regeneration in response to disease and injury. This mouse expresses a Cre-dependent firefly luciferase gene and an estrogen-responsive Cre-recombinase under the control of the Pax7 locus. Because satellite cells are the only muscle cells in the adult that express Pax7, these mice 